\title{
A ten-year follow-up of haemorrhagic proctitis
}

\author{
A. MYers \\ M.B., M.R.C.P.
}

\author{
D. M. HUMPHREYS \\ M.B., M.R.C.P.
}

\author{
E. V. Cox \\ M.D., F.R.C.P. \\ Department of Medicine, Royal Berkshire Hospital, Reading
}

\begin{abstract}
Summary
Seventy-four cases of haemorrhagic proctitis have been observed during a 10-year period. Inheritance, psychological stress and constipation may be of aetiological significance. A graded therapeutic effect using roughage, salazopyrin and local steroids emphasized the necessity of using all those forms of therapy, both to obtain and maintain remission. Peripheral complications and persisting abnormal serum protein patterns were associated with a high incidence of progression of the disease beyond the confines of the rectum.
\end{abstract}

\section{Introduction}

HAEMORRHAGIC proctitis is an inflammatory process of the rectal mucosa with no specific cause. The changes are indistinguishable from those seen in ulcerative colitis but are limited sigmoidoscopically to the rectum. The colon is normal radiologically. Thus the disease is limited to the rectum and at sigmoidoscopy there is a clear demarcation level as indicated by normal mucosa never higher than $20 \mathrm{~cm}$ above the anus. Only a small percentage of patients progress to ulcerative colitis (Farmer and Brown, 1966; Truelove, 1959; Sparberg, 1968). As a result of this, it has been suggested that it is a discrete entity although it is generally concluded that it is a variant of ulcerative colitis. The data presented here are derived from a prospective and prolonged study of patients with haemorrhagic proctitis. The benign and the relapsing nature of the condition is emphasized, and the effects of a routine therapeutic approach have been analysed. From a prognostic point of view it seems justifiable to separate it clinically from ulcerative colitis.

\section{Materials and methods}

All the seventy-four patients in this study were originally referred to the Gastroenterology clinic at the Royal Berkshire Hospital. The sigmoidoscopic

Address for reprints: Dr A. Myers, 102 Cavendish Drive, Leytonstone, London E.11. and radiological findings showed the presence of 3 an inflammatory disorder limited to the rectum. in Specific infections and disease elsewhere in the gastrointestinal tract were excluded by detailed 8 investigation at the time of diagnosis. The investiga- in tion was limited to those patients who were per- $\triangle$ manently resident in this area and who were willing 을 to attend regularly for follow-up.

\section{Therapeutic approach \\ Therapy $I$}

All patients were advised to take a normal diet and the roughage or fibre content was supplemented with either Isogel or Prewett's Bran. All patients were advised to avoid any constipating agents and if they suffered from constipation, mild aperients were advised.

\section{Therapy II}

To Therapy I, salazopyrin $1.5-3.0 \mathrm{~g}$ daily was added if rectal bleeding was a persistent feature. This was replaced with erythromycin if the patient was allergic to salazopyrin.

\section{Therapy III}

Predsol (prednisolone sodium phosphate ester) enemas or suppositories were added to Therapy II if rectal bleeding was severe and if symptoms failed to respond to Therapy II.

As patients responded to treatment, it was hoped to reduce the therapy from III to II and then from II to I. However, this did not occur consistently, usually because of the whim of the patient, and $\sigma$ occasionally because of alternative advice from the general practitioner.

\section{Relapses}

Relapse was defined as a recurrence of symptoms of haemorrhagic proctitis with a return of rectal bleeding. The time of onset of relapse and the time of recovery were noted, as were any apparent precipitating factors. It soon became apparent that sigmoidoscopic appearances were unsatisfactory in 
determining relapses as by the time some patients were reviewed, sigmoidoscopic appearances had obviously improved and in other instances quite severe sigmoidoscopic changes were present in remission.

\section{Follow up}

This varied from 3 to 122 months with an average of 45 months.

\section{Clinical findings at presentation}

Thirty-nine patients were male $(52 \cdot 7 \%)$ and thirtyfive female $(45.3 \%)$. Ages at the time of the study ranged from 18 to 72 years with an average of 40.8 years. The age of onset of the disease varied between 16 to 70 years with an average of 35.5 years.

\section{Initial symptoms}

Sixty-six patients $(89.5 \%)$ had an alteration in large bowel habit. It was increased in frequency in 59 $(80 \%)$ and reduced in frequency in $7(9.5 \%)$. Episodes of abdominal pain occurred in $56.8 \%$; blood mixed with faeces was passed by $37.8 \%$; blood and mucus passed by $56.8 \%$; two had mucus only and two had neither blood nor mucus. There was a past history of constipation in thirty-six patients $(48.6 \%)$, one of diarrhoea preceding their current bowel problems in twenty-one. Tense and anxious personalities were noted in fifty-two. Only five were recorded as suffering from migraine.

\section{Local complications at presentation}

The incidence of local complication was very low. One patient had an anal fissure and one a rectal prolapse.

\section{Clinical findings}

There was clinical evidence of iron deficiency (koilonychia or angular stomatitis) in nine patients $(12.2 \%)$. Five patients had lost weight. One patient had pigmentation and phlyctenular conjunctivitis. One had erythema nodosum and one ankylosing spondylitis with peripheral joint disease.

\section{Family history}

There was a high incidence of ulcerative colitis in the families of the patients reviewed. Five cases had one or more parents or siblings affected. One case had a family history of Crohn's disease. There was a family history of unspecified bowel disorder in five patients. Cancer of the large bowel was not recorded.

\section{Initial investigations \\ Haematology}

A mild degree of anaemia was common, twentyfive patients $(33 \%)$ had a haemoglobin below 12.5 $\mathrm{g} / 100 \mathrm{ml}$ and ten of these were below $10 \mathrm{~g} / 100 \mathrm{ml}$. A raised ESR was more common. An ESR over 20 $\mathrm{mm} / \mathrm{hr}$ was observed in $40 \%$ although in only $17 \%$ was it over $29 \mathrm{~mm} / \mathrm{hr}$.

\section{Plasma proteins}

The serum albumin level was consistently above $3.5 \mathrm{~g} / 100 \mathrm{ml}$. The electrophoretic pattern was normal in forty-seven and remained so in thirty-eight of these. Twenty-seven cases $(36.5 \%)$ had abnormal electrophoretic pattern of the plasma proteins. The $\gamma$-globulin was elevated in fifteen patients and reduced in one. The $\alpha_{2}$-globulin was elevated in sixteen patients, five of these having associated $\beta$-globulin elevations. Of the forty-seven patients with normal plasma proteins initially, thirty-eight remained unchanged. Transient abnormality developed in three patients $(4.3 \%)$ and permanent abnormalities developed in six. Of the twenty-seven patients with abnormal plasma proteins initially, sixteen became normal and remained so. Abnormality was permanent in eleven patients.

\section{Barium enema}

In spite of the high incidence of diarrhoea and preparation with oral bisacodyl followed by bisacodyl suppositories, thirty-one patients had evidence of faecal retention and were reported by the radiologist to be poorly prepared. Of these, twenty-four showed both proximal and distal retention, five proximal only, and two distal retention only. There was no evidence of ulceration or any other abnormality on X-rays.

\section{Changes of sigmoidoscopy}

At some stage, all the patients showed erythema and granularity. Bleeding was observed in all but twelve patients and in none of these was progression of the disease observed. The disease extended to approximately $20 \mathrm{~cm}$ in $75 \%$ of the patients.

\section{Therapy before being seen initially}

Approximately one third (twenty-four) had not received previous treatment; another third had taken constipating agents (codeine and opiate-containing mixtures) (this number was probably higher, and probably included antibiotics with solidifying agents). The remainder received roughage supplements and salazopyrin, particularly as the regimen became known in the area. In the patients receiving constipating agents, this therapy had preceded rectal bleeding in the high proportion of cases.

\section{Initial treatment at the Royal Berkshire Hospital}

Twelve patients were commenced on, and responded to, Therapy I. All but one required subsequent treatment with either Therapy II or III. 
Therapy II was the initial successful treatment in forty-one patients, but twenty-two of these subsequently required Therapy III to treat relapses.

Therapy III was used from the beginning in twenty patients, partly because a number of these had already been treated with Therapy II by their general practitioner, and partly because of the severity of their symptoms.

In summary, $98 \%$ required Therapy II at some stage in their treatment and $74 \%$ required the addition of topical steroids (Therapy III).

\section{Therapy required to obtain the first remission of symptoms}

Therapy $I$ in four patients; II in thirty-three; III in thirty-seven.

\section{Length of time to obtain a remission}

Twenty-three patients $(31 \%)$ remitted within 1 month of starting therapy at the Royal Berkshire Hospital; thirty-two remitted within 1-3 months, and the average length of time taken to obtain a remission was 3.4 months. As would be expected, the length of time to achieve remission was longer in those patients who progressed from Therapy I to Therapy II and subsequently to Therapy III, and in those who commenced on Therapy II and progressed to Therapy III.

\section{Relapses}

Eighteen patients $(24 \%)$ had no relapse during the period of follow-up of which the average time was 2.1 years (range 4-74 months). Seventeen patients had one relapse during the follow-up period which averaged 3.3 years (range 3-94 months). Twenty patients had two relapses during an average followup period of 3.9 years (range 10-108 months). Ten patients had three relapses (range of follow-up 31-90 months) and nine had more than three relapses during a follow-up period ranging from 48 to 104 months. The maximum number of relapses was six, occurring in two patients and they were followed-up for 78 months and 106 months respectively. Thus, the longer the period of follow-up, the greater the likelihood of relapse.

Forty patients $(48.3 \%$ ) relapsed for the first time within 1 year of the first remission, and eight $(10.8 \%)$ relapsed between 1 and 2 years. The average length of time between the beginning of follow-up and the first relapse was $11 \cdot 6$ months.

\section{Therapy at the time of first relapse}

Fifty-six patients had one or more relapses, but eleven of these were not taking any therapy at the time of relapse; all had stopped treatment of their own volition and relapsed within 3 months of stopping it. The average length of remission in this group was 13 months (range 1-46 months). One $\frac{2}{3}$ patient had been on Therapy I, six on Therapy II and $\stackrel{\otimes}{\varrho}$ four on Therapy III.

Eighteen patients relapsed while taking Therapy $\mathrm{I} ; \overrightarrow{\overrightarrow{\vec{F}}}$ three of these had recently discontinued Therapy II $\stackrel{+}{+}$ and nine had reduced Therapy III to Therapy I. The average length of remission in this group was $15 \cdot 8 \frac{\bar{\sigma}}{\mathrm{N}}$ months (range 2-38 months). Twenty-two patients $\frac{\pi}{\sigma}$ relapsed while taking Therapy II, and nine of these $\odot$ had been on Therapy III. The average length of $ळ$ remission in this group was 10 months (range 1-54 $\vec{\circ}$ months).

The remainder relapsed while on Therapy III. $\overrightarrow{\vec{\omega}}$ The average length of remission was 2.8 months (range 1-5 months). It is of interest that twenty patients relapsed while taking the therapy that $\frac{3}{\mathrm{~F}}$. initially induced remission. In summary, two $(3.6 \%)$ i of the fifty-six patients who relapsed, did so when 8 Therapy I was discontinued and the remainder o relapsed on reducing either Therapy II or Therapy N III. It is noteworthy that relapse was preceded by constipation in twenty-six patients $(46.9 \%$ of all relapses) and thirty-two $(57 \cdot 1 \%$ of all relapses) $\vec{\nabla}$ relapsed at a time of well defined psychological stress.

\section{Multiple relapses \\ The average length of time between first and} second, and second and third relapses was fair consistent at $1 \cdot 1$ and 1.3 years respectively. Of thos thirty-nine patients who had more than one relapse, only six $(15 \%)$ did so on Therapy III. Twenty-one patients $(54 \%)$ were on Therapy II, the remainder $\stackrel{\odot}{\varnothing}$ were on Therapy $I$.

\section{Therapy at time of review}

No treatment in seven; bulk only (Therapy I) in twenty-three; bulk and antibiotics (Therapy II) in thirty; bulk, antibiotics and steroids (Therapy III) in fourteen.

At the time of review, seventeen $(23 \%)$ patients had had a prolonged period of remission of symp- $\delta$ toms and had rectal mucosa of normal appearance. The average length of remission was 2.6 years. 은 Forty-six patients $(62.2 \%)$ remained symptom-free but had abnormal rectal mucosa as seen at sigmoidoscopy, with an average remission of 1.4 years. In most of these cases the appearance of the rectal o mucosa had improved from that seen at initial 0 sigmoidoscopy. The sigmoidoscopy performed at $\underset{\mathrm{W}}{\mathrm{N}}$ the time of the review was as follows: Normal, seventeen $(23 \%)$; greatly improved, twenty $(27 \%) ; 0$ improved, twenty-seven $(36.4 \%)$; unchanged, four $\frac{C}{\Phi}$ $(5 \cdot 4 \%)$; worse, three $(4 \%)$.

Progression to ulcerative colitis and procto-sigmoiditis In seven patients the disease spread beyond the 
rectum. In two, the disease process remained localized to the rectum and sigmoid colon but in the other five patients the disease spread to involve part or all of the colon. The two patients in whom the disease remained localized to the rectum and sigmoid colon remitted on Therapy III with bulk, antibiotics and local steroids.

Of the five cases in the series who progressed to ulcerative colitis, the average age was 39.4 years (range of 25-72 years). The age of onset of symptoms of proctitis ranged from 16-63 years with an average of 31.4 years. Of these, two were male, and three female. The time from first onset of symptoms of haemorrhagic proctitis to the diagnosis of ulcerative colitis varied from 78 to 112 months with an average of 93.2 months. During review, three of the cases which developed ulcerative colitis either had or developed peripheral complications known to be associated with ulcerative colitis. One had erythema nodosum and phlyctenular conjunctivitis at presentation; one developed peripheral joint disease; one developed episcleritis and erythema nodosum during the follow-up. Weight loss and iron deficiency were present in three of the five patients in whom the disease progressed. Only one other patient with disease limited to the rectum developed peripheral complications (ankylosing spondylitis with peripheral joint disease).

Of these patients developing ulcerative colitis, the plasma protein electrophoresis was initially persistently abnormal in one; three developed permanently abnormal plasma proteins and one developed abnormal plasma proteins which returned to normal with remission of the disease. In one of the patients who developed procto-sigmoiditis, abnormal plasma proteins were present initially and they remained abnormal during the time of follow-up. In the other patient, the plasma protein electrophoresis was persistently normal. Persistently abnormal plasma proteins were noted in eleven patients in whom the disease remained limited to the rectum. The follow-up of this group was on average 53.8 months (range 10-90 months). It is conceivable that if this group had been followed-up for as long as the group that progressed to ulcerative colitis, further cases of progression of the disease would have come to light. In one of the cases that developed into ulcerative colitis, a colectomy was necessary. In the other four the disease went into remission.

\section{Discussion}

The age, sex, initial symptomatology and clinical findings, including sigmoidoscopy and barium enema, do not differ significantly from those of other authors (Sparberg, 1968; Farmer and Brown, 1966, 1971, 1972; Wilson, 1970; Cropper, 1955; Truelove, 1959; Newell and Avery-Jones, 1958; Freiberger and
Muller-Wieland, 1968). The disease is benign in that there were no deaths in this series and few peripheral complications. However, in spite of continued follow-up with a set therapeutic regime, only $24.3 \%$ of patients had no recurrence, and the follow-up in this group was short $(2 \cdot 2$ years). The disease usually follows a relapsing course. The longer the follow-up period, the more likely the chance of relapse. In those who relapsed more than once, the relapses occurred at approximately yearly intervals. From the aetiological point of view, a high family incidence of chronic inflammatory disease of the large bowel has been observed. Also $37.1 \%$ were recorded as relapsing at the time of well defined psychological stress, and $72 \%$ had tense and anxious personalities. Freiberger and Muller-Wieland observed that psychological stress played a part in the incidence of relapses. The observations that $42 \%$ of patients had faecal retention at their initial barium enema, $47 \%$ of relapses were preceded by constipation, and a high proportion of patients had been treated with constipating agents in many instances before the initial onset of rectal bleeding, suggested that constipation may be a precipitating factor. However, a diet with added roughage or fibre content, although reducing constipation, was insufficient therapy to induce remission or to maintain remission once it had been induced. In fact, all but one of the patients required either salazopyrin or erythromycin at some time, and $73 \%$ required topical steroids. As many as $37 \%$ required Therapy III (bulk, antimicrobials and local steroids) to induce the first remission. The prophylactic value of antibiotics and steroids was emphasized by the relapses of thirty-six patients after cessation of antibiotics or steroids. The better prophylactic effect of steroids as compared with antibiotics is suggested by the observation that twenty-four relapsed when steroids were discontinued and only ten when anti-microbials were stopped. This would suggest that the best way to induce remission and to maintain it is with a combination of bulk, antibiotics and steroids. This is contrary to the views of Sparberg (1968) who felt that aggressive therapy was of little value as attacks were short lived and self limited. Farmer and Brown (1966) and Truelove (1959) recommended a short course of local steroids in addition to antibiotics.

At the time of final review only $23 \%$ of patients could be considered cured as they had normal sigmoidoscopic appearances and were symptom free. However, during the course of follow-up three patients were observed who were apparently cured clinically and sigmoidoscopically and yet relapses occurred after symptomless periods of between 3 and 4 years. Thus, it would appear that life-long followup is mandatory. Since the majority of patients $(62.2 \%)$ who were symptom free still had abnormal 
sigmoidoscopic appearances, their continued followup is obviously necessary. Also, from the therapeutic point of view, reducing therapy with persisting sigmoidoscopic changes is most probably unwise, especially with regard to the high relapse rate which follows such a reduction. This indication only became apparent during the preparation of this review.

In seven patients $(9.4 \%)$ the disease progressed beyond the rectum: two of these remained localized to the sigmoid and in the other five the disease spread to the whole part of the colon. This agrees with the progression rate quoted by other authors. Sparberg (1961) gave a figure of $8.3 \%$ in a 5 -year follow-up, $22 \%$ in a $5-10$ year period and $25 \%$ in a small number followed up for more than 15 years. Farmer and Brown (1971) gave a figure of $5.9 \%$ for fifty-one patients with an average follow-up time of 6 years. Wilson (1970) gave a figure of $5.2 \%$ in fourteen cases over a period of 3 years. Truelove (1959) saw no progression in twenty-two patients followed-up for only 9 months. Apart from one case of peripheral joint disease which may have been coincidental, the only complications occurring in those that continued with localized rectal disease were iron deficiency and weight loss. Other peripheral complications observed (erythema nodosum, pigmentation, episcleritis and ankylosing spondylitis) all occurred in those in whom the disease did not remain confined to the rectum. All these complications are those that have been regularly reported as occurring in ulcerative colitis. In only one paper (Hardy-Smith and McPhee, 1971) have comments been made on the plasma protein electrophoresis in haemorrhagic proctitis. In that limited series no patient with haemorrhagic proctitis had abnormal plasma protein electrophoresis. The prognostic value of the plasma proteins has long been recognized in the author's unit. In six of the seven cases in whom the disease spread beyond the rectum, all either started with abnormal proteins $\cong$ or developed them during follow-up. The prognostic 3 value is only relevant if the proteins are monitored $\stackrel{\varnothing}{\Omega}$ during the course of the illness since a significant $C$ proportion of the series $(36.5 \%)$ presented with abnormal proteins but only one of these cases progressed to ulcerative colitis. The most significant feature was a change from normality to permanent $\frac{\bar{c}}{\bar{c}}$ abnormality, indicating a progression to ulcerative $\vec{\nabla}$ colitis. The follow-up period of eleven patients with $\propto$ permanently abnormal plasma proteins with disease $\approx$ limited to the rectum was, on average, almost 3 years $\vec{\circ}$ less than those in whom ulcerative colitis occurred, and they may therefore subsequently develop $\vec{\omega}$ progressive disease.

\section{References}

CROPPER, C.F.J. (1955) Idiopathic granular proctitis. Lancet, i, 1253.

FARMER, G.R. \& BRown, C.H. (1966) Ulcerative proctitis iN course and prognosis. Gastroenterology, 51, 219.

FARMER, G.R. \& Brown, C.H. (1971) Course and prognosis 0 of ulcerative proctitis. American Journal of Gastroentero$\log y, 56,227$.

FARMER, G.R. \& BROWN, C.H. (1972) Emerging concepts of $\frac{D}{2}$

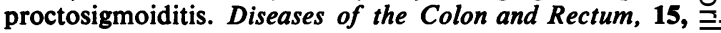
142.

Freiberger, H. \& Muller-Weiland, K. (1968) Proct 8 sigmoiditis-a special form of chronic ulcerative colitis American Journal of Proctology, 194, 270.

HARDY-SMITH, A. \& MCPheE, I.W. (1971) A clinico-immun pathological study of ulcerative colitis and ulcerative proctitis. Gut, 12, 20.

Newell, A.C. \& AVERY-Jones, F. (1958) Observations on the management of idiopathic proctitis. Proceedings of the $\frac{\mathrm{D}}{\mathrm{Q}}$ Royal Society of Medicine, 51, 431.

SPARBERG, M. (1968) Ulcerative proctitis. Texas Medical $\overrightarrow{\vec{\sigma}}$ Journal, 64, 52.

TRUELOVE, S.C. (1959) Suppository treatment of haemorrhagic proctitis. British Medical Journal, 1, 955.

WILsON, E. (1970) Salazopyrin in the treatment of granular proctitis. Australian and New Zealand Journal of Surgery, $\bar{\partial}$ 39, 275. 